\title{
openheart NOACs versus warfarin for stroke prevention in patients with AF: a systematic review and meta-analysis
}

\author{
Tim Hicks, ${ }^{1}$ Fiona Stewart, ${ }^{2}$ Anne Eisinga ${ }^{3}$
}

To cite: Hicks T, Stewart $F$, Eisinga $A$. NOACs versus warfarin for stroke prevention in patients with $\mathrm{AF}$ : a systematic review and meta-analysis. Open Heart 2016;3:e000279. doi:10.1136/openhrt-2015000279

- Additional material is available. To view please visit the journal (http://dx.doi.org/ 10.1136/openhrt-2015000279).

Received 10 April 2015 Revised 11 December 2015 Accepted 14 December 2015

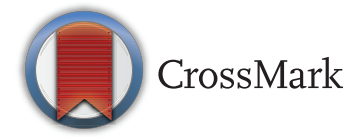

\footnotetext{
${ }^{1}$ Dorset County Hospital, Dorchester, UK

${ }^{2}$ Health Services Research Unit, University of Aberdeen, Aberdeen, UK

${ }^{3}$ UK Cochrane Centre, Oxford, UK
}

Correspondence to Dr Tim Hicks;

tim.hicks.09@aberdeen.ac.uk

\section{ABSTRACT}

Background: Warfarin has been the anticoagulant of choice for the prevention of ischaemic stroke in patients with atrial fibrillation (AF). Novel oral anticoagulants (NOACs) are increasingly used as an alternative.

Objectives: The objective of this review was to evaluate the efficacy and safety of the NOACs versus warfarin in patients with AF.

Search methods: Medline, EMBASE and grey literature search for all phase II and III randomised control trials.

Data collection/analysis: Two authors independently reviewed abstracts and performed data extraction of eligible full-text articles. Revman V.5 was used for meta-analysis.

Main results: 12 studies were identified with a total study population of 77011 . NOACs demonstrated a reduction in the composite of stroke or systemic embolic events OR 0.85 ( $95 \% \mathrm{Cl} 0.75$ to 0.98$)$, a $52 \%$ reduction in intracranial haemorrhage $0 \mathrm{R} 0.48(95 \% \mathrm{Cl}$ 0.40 to 0.57 ) and a $14 \%$ reduction in mortality $\mathrm{OR}$ 0.86 (0.82 to 0.91$)$. The 30 -day end of study switch to warfarin demonstrated an in increase in stroke or systemic embolic events OR $2.60(95 \% \mathrm{Cl} 1.61$ to 4.18) and an increase in major bleeding OR 2.19 (95\% $\mathrm{Cl} 1.42$ to 3.36 ).

Conclusions: NOACs are superior to warfarin for the prevention of the composite of stroke and systemic embolism in patients with AF and an additional risk factor for stroke. There is a significant reduction in intracranial haemorrhage, which drives the finding of significantly lower mortality. During the poststudy switch from NOACs to warfarin there is an excess of the composite of stroke and systemic embolism as well as major bleeding events, which may be of significance in clinical practice.

\section{INTRODUCTION}

\section{Description of the condition}

Atrial fibrillation is a common arrhythmia with a less efficient passage of blood through the atria with the potential for stasis and increased risk of thrombus formation particularly in the atrial appendage. This thrombus formation gives rise to the potential for embolism, which can manifest as ischaemic stroke. ${ }^{12}$

\section{KEY QUESTIONS}

What is already known about this subject?

- Factor Xa inhibitors and direct thrombin (factor Ila) inhibitors, novel oral anticoagulants (NOACs) are effective in the prevention of ischaemic stroke in patients with atrial fibrillation and at least one additional risk factor for stroke when compared to warfarin.

What does this study add?

- This is an independent meta-analysis of all phase II and III clinical trials with 77011 participants, which additionally demonstrates a reduction in the composite of stroke and systemic embolism, a halving of the number of haemorrhagic strokes, which drives a reduction in all-cause mortality.

- During the 30-day poststudy switch from NOACs to warfarin there is an increase in the composite of stroke and systemic embolism as well as major bleeding.

How might this impact on clinical practice?

- NOACs could progress to being the first-line therapy for stroke prevention in patients with atrial fibrillation and at least one additional risk factor for stroke.

- Thromboembolic and bleeding events within 30 days of discontinuation of NOACs and switch to warfarin should be considered as adverse events and highlighted through the reporting system of the clinician's country of practice.

\section{Description of control/comparison}

Warfarin is a vitamin $\mathrm{K}$ antagonist, which exerts its anticoagulant effect by inhibiting the clotting factors II, VII, IX and X. Regrettably, it is, however, prone to numerous drug and food interactions, ${ }^{3}$ which necessitates regular blood testing to maintain the international normalisation ratio (INR) within the therapeutic range. Significant patient time and medical resources are required and the effective vigilance of medical practitioners in the bespoke tailoring of warfarin dose to the individual is far from straight forward. 


\section{Description of the intervention and how it might work}

Novel oral anticoagulants (NOACs) have a more specific mode of action directly targeting just one clotting factor. The factor Xa inhibitors and direct thrombin (factor IIa) inhibitors produce a more predictable and less labile anticoagulant effect, which is less susceptible to drug and food interactions and do not require regular monitoring.

\section{Why it is important to do this review}

There have been several reviews addressing similar interventions and outcomes, ${ }^{4-6}$ however, this review is an independent review, which aimed to identify all NOACs with high-quality phase II and III trials. In addition this review evaluated data from the end of the clinical trials when NOACs are stopped and participants are switched to warfarin. This has mostly gone unreported and is a key element of understanding the place of NOACs and particularly relevant considering the extent of discontinuation of around $20-24 \%$ in the clinical trial setting.

This review also applied the Grading of Recommendations, Assessment, Development and Evaluation (GRADE) criteria for assessing the strength of evidence in a summary of findings table.

\section{Objectives}

1. To evaluate the efficacy and safety of the NOACs with warfarin as a comparator as well as the end of trial 'switch' to warfarin in patients with atrial fibrillation and an additional risk factor for stroke.

2. To formulate a GRADE summary of findings table.

\section{METHODS}

The review was conducted in accordance with PRISMA guidelines and an a priori protocol for the review was registered with Prospero. ${ }^{7}$

\section{Literature search, eligibility criteria and data collection}

Highly sensitive electronic searches were conducted to identify randomised control trials comparing NOACs to warfarin. The databases searched were: MEDLINE (1946 to March week 3 2014), MEDLINE-In-Process and other non-indexed Citations(31 March 2014), Embase (OvidSP 1974 to 2014 April 01, The Cochrane Library (Issue 4, April 2014), Cochrane Central Register of Controlled Trials (Issue 1, January 2014), Database of Abstracts of Reviews of Effects (Issue 1, January 2014), Latin American and Caribbean Health Sciences (LILACS) (April 2014) and Scopus (April 2014). No language or date restrictions were imposed on the search. Reference lists of included studies were checked to identify further relevant articles. Additionally, recent conference proceedings of the European Society of Cardiology, European Haematology Association and American Society of Hematology and websites of pharmaceutical manufacturers were searched. Study authors were contacted to request additional data or to seek clarification where information in the published report was lacking or ambiguous.

Two reviewers independently screened the titles and abstracts identified by the literature search. Full-text papers selected from the search results were also independently screened by two reviewers. Any disagreements were resolved by consensus. After developing and piloting a data extraction form, data were extracted from the included studies by one reviewer and checked by a second.

Quality assessment was carried out using the Cochrane Collaboration's tool for assessing risk of bias (ROB) (see online supplementary appendix figures 3 and 4). The Grading of Recommendations Assessment, Development and Evaluation (GRADE) evidence quality assessment tool was used to assess outcomes across studies.

\section{Statistical analysis}

Data were imported into Review Manager V.5.2 (The Cochrane Collaboration, Oxford) for quantitative synthesis. Dichotomous outcomes were converted from HRs or risk ratios (RR) to ORs, with 95\% CIs. Heterogeneity between studies was assessed by visual inspection of plots of the data, the $\chi^{2}$ test for heterogeneity, and the $\mathrm{I}^{2}$ statistic. $^{8}$

Fixed effects meta-analyses were undertaken where statistical heterogeneity was judged to be low. Where statistical heterogeneity was moderate to high, random effects meta-analyses were undertaken to take into account within study and between study variability.

When included studies had multiple intervention arms comparing different doses of NOAC $\mathrm{s}$ with a single comparison arm of warfarin, the smaller phase II study the data for the NOACs arms was combined to produce a single summary statistic for comparison with the warfarin arm. For larger phase III studies with more than one dose of NOACs, the number of participants and events in the warfarin arms were divided evenly into the same number of corresponding NOACs arms. This ensures that multiplicity of data does not unduly influence the pooled effect estimate in the forest plot.

\section{SUMIMARY OF RESULTS Studies identified}

Twelve studies along with eight further reports ${ }^{9-28}$ were identified which were suitable for meta-analysis (see online supplementary appendix figure 1 and 2). All were drug company funded. Three were large international multicentre phase III trials of factor Xa inhibitors, apixaban, rivaroxaban and edoxaban, one was a smaller study of rivaroxaban with Japanese participants. All four of studies were high quality low ROB trials with double blind intervention arms and warfarin control arms. Outcome assessment was also blinded. There was one large phase III trial of the direct thrombin (factor IIa) inhibitor dabigatran. 


\begin{tabular}{|c|c|c|c|c|c|c|}
\hline \multirow[t]{2}{*}{ Outcome } & \multicolumn{2}{|c|}{$\begin{array}{c}\text { Novel Oral } \\
\text { Anticoagulants }\end{array}$} & \multicolumn{2}{|c|}{ Warfarin } & \multirow[t]{2}{*}{ Model } & \multirow[t]{2}{*}{$\begin{array}{l}\text { Odds Ratio } \\
\mathrm{M}-\mathrm{H} 95 \% \mathrm{Cl}\end{array}$} \\
\hline & Events & Total $\mathrm{n}=$ & Events & Total $n=$ & & \\
\hline Stroke or Systemic Embolic Event & 1498 & 44966 & 1135 & 30528 & Fixed Effects & $0.87(0.81-0.95)$ \\
\hline Stroke or Systemic Embolic Event & 1498 & 44966 & 1135 & 30528 & Random Effects & $0.85(0.75-0.98)$ \\
\hline Ischaemic Stroke & 1168 & 45504 & 736 & 30846 & Fixed Effects & $1.02(0.93-1.12)$ \\
\hline Ischaemic Stroke & 1168 & 45504 & 736 & 30846 & Random Effects & $0.98(0.82-1.16)$ \\
\hline Intracranial Haemorrhage & 207 & 43921 & 308 & 30197 & Fixed Effects & $0.48(0.40-0.57)$ \\
\hline Mortality & 3317 & 44866 & 2431 & 30568 & Fixed Effects & $0.86(0.82-0.91)$ \\
\hline $\begin{array}{l}\text { Major or Clinically Relevant Non- } \\
\text { Major Bleeding }\end{array}$ & 5022 & 33413 & 4246 & 24767 & Fixed Effects & $0.81(0.77-0.84)$ \\
\hline $\begin{array}{l}\text { Major or Clinically Relevant Non- } \\
\text { Major Bleeding }\end{array}$ & 5022 & 33413 & 4246 & 24767 & Random Effects & $0.84(0.68-1.03)$ \\
\hline Major Bleeding & 2180 & 45755 & 1826 & 30839 & Fixed Effects & $0.78(0.73-0.83)$ \\
\hline Major Bleeding & 2180 & 45755 & 1826 & 30839 & Random Effects & $0.76(0.62-0.93)$ \\
\hline Gastrointestinal Bleeding & 1012 & 42943 & 598 & 29850 & Fixed Effects & $1.12(1.01-1.25)$ \\
\hline Gastrointestinal Bleeding & 1012 & 42943 & 598 & 29850 & Random Effects & $1.05(0.82-1.36)$ \\
\hline Myocardial Infarction & 698 & 44661 & 446 & 30505 & Fixed Effects & $1.02(0.90-1.15)$ \\
\hline Myocardial Infarction & 698 & 44661 & 446 & 30505 & Random Effects & $1.03(0.881 .20)$ \\
\hline
\end{tabular}

Pooled Results 30 day end of study switch to warfarin

\begin{tabular}{|c|c|c|c|c|c|c|}
\hline \multirow[t]{2}{*}{ Outcome } & \multicolumn{2}{|c|}{$\begin{array}{c}\text { Novel Oral } \\
\text { Anticoagulants }\end{array}$} & \multicolumn{2}{|c|}{ Warfarin } & \multirow[t]{2}{*}{ Model } & \multirow[t]{2}{*}{$\begin{array}{l}\text { Odds Ratio } \\
\mathrm{M}-\mathrm{H} 95 \% \mathrm{Cl}\end{array}$} \\
\hline & Events & Total $n=$ & Events & Total $n=$ & & \\
\hline Stroke or Systemic Embolic Event & 68 & 28282 & 22 & 21107 & Fixed Effects & $2.60(1.61-4.18)$ \\
\hline Major Bleeding & 79 & 27590 & 28 & 20446 & Fixed Effects & $2.19(1.42-3.36)$ \\
\hline
\end{tabular}

Figure 1 Table of pooled results.

\begin{tabular}{|c|c|c|c|c|c|c|c|}
\hline \multirow[b]{2}{*}{ Study or Subgroup } & \multicolumn{2}{|c|}{ Novel Oral Anticoagulant } & \multicolumn{2}{|c|}{ Walfarin } & \multirow[b]{2}{*}{ Weiglit } & \multirow{2}{*}{$\begin{array}{l}\text { Odds Ratio } \\
\text { M-H. Random. } 95 \% \mathrm{Cl}\end{array}$} & \multirow{2}{*}{$\begin{array}{l}\text { Odds Ratio } \\
\text { M-H, Random, } 95 \% \mathrm{Cl}\end{array}$} \\
\hline & Events & Total & Events & Total & & & \\
\hline \multicolumn{8}{|c|}{ 1.1.1 Phase III Trials of F actor Xa Inhibitors } \\
\hline ARISTOTLE 2011 & 212 & 9210 & 265 & 9081 & $17.3 \%$ & $0.78[0.65,0.94]$ & 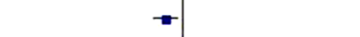 \\
\hline ROCKET AF 2011 & 269 & 7081 & 306 & 7090 & $18.2 \%$ & $0.88[0.74,1.03]$ & \\
\hline ENGAGE 2013 Edoxaban 30 & 383 & 7034 & 168 & 3518 & $17.1 \%$ & $1.15[0.95,1.38]$ & - \\
\hline ENGAGE 2013 Edoxaban 60 & 296 & 7035 & 169 & 3518 & $16.6 \%$ & $0.87[0.72,1.06]$ & \\
\hline J-Rocket AF 2012 & 11 & 637 & 22 & 637 & $3.0 \%$ & $0.49[0.24,1.02]$ & \\
\hline Subtotal $(95 \% \mathrm{Cl})$ & & 30997 & & 23844 & $722 \%$ & $0.88[0.75,1.04]$ & \\
\hline Total events & 1171 & & 930 & & & & \\
\hline \multicolumn{8}{|c|}{$\begin{array}{l}\text { Heterogeneity: } \operatorname{Tau}^{2}=0.02 ; \mathrm{Ch}^{2}=11.62, \mathrm{df}=4(P=0.02) ; \mathrm{I}^{2}=66 \% \\
\text { Test for overall effect: } Z=1.45(P=0.15)\end{array}$} \\
\hline \multicolumn{8}{|c|}{ 1.1.2 Phase III Trials of Direct Thrombin (Factor lla) Inhibitors } \\
\hline RE-LY 2009 Dabigatran 110 & 183 & 6015 & 101 & 3011 & $13.6 \%$ & $0.90[0.71,1.16]$ & \\
\hline RE-LY 2009 Dabigatran 150 & 134 & 6076 & 101 & 3011 & $12.9 \%$ & $0.65[0.50,0.84]$ & \\
\hline Subtotal $(95 \% \mathrm{CI})$ & & 12091 & & 6022 & $26.5 \%$ & $0.77[0.56,1.06]$ & \\
\hline Total events & 317 & & 202 & & & & \\
\hline \multicolumn{8}{|c|}{$\begin{array}{l}\text { Heterogeneity: } \text { Tau }^{2}=0.04 ; \mathrm{Chi}^{2}=3.23, \mathrm{df}=1(\mathrm{P}=0.07) ; \mathrm{I}^{2}=69 \% \\
\text { Test for owerall effect: } Z=1.59(\mathrm{P}=0.11)\end{array}$} \\
\hline \multicolumn{8}{|c|}{ 1.1.3 Phase II Trials of Factor Xa Inhibitors } \\
\hline Edoxaban USiEurope 2010 & 6 & 893 & 3 & 250 & $0.9 \%$ & $0.56[0.14,2.24]$ & \\
\hline $\begin{array}{l}\text { OPAL- } 12010-\text { YM } 150 \\
\text { Subtotal }(95 \% \text { Cl })\end{array}$ & 2 & $\begin{array}{r}354 \\
1247\end{array}$ & 0 & $\begin{array}{r}94 \\
344\end{array}$ & $\begin{array}{l}0.2 \% \\
1.1 \%\end{array}$ & $\begin{array}{r}1.34[0.06,28.16] \\
0.65[0.18,2.30]\end{array}$ & \\
\hline Total events & 8 & & 3 & & & & \\
\hline \multicolumn{8}{|c|}{$\begin{array}{l}\text { Heterogeneity: } \text { Tau }^{2}=0.00 ; \mathrm{Chi}^{2}=0.27, \mathrm{df}=1(\mathrm{P}=0.61) ; \mathrm{I}^{2}=0 \% \\
\text { Test for overall effect: } Z=0.67(\mathrm{P}=0.50)\end{array}$} \\
\hline \multicolumn{8}{|c|}{ 1.1.4 Phase II Trials of Direct Thrombin (Factor Ila) Inhibitors } \\
\hline $\begin{array}{l}\text { Lip } 2009-A Z D 0837 \\
\text { Subtotal }(\mathbf{9 5} \% \mathrm{Cl})\end{array}$ & 2 & $\begin{array}{l}631 \\
631\end{array}$ & 0 & $\begin{array}{l}318 \\
318\end{array}$ & $\begin{array}{l}0.2 \% \\
0.2 \%\end{array}$ & $\begin{array}{r}2.53[0.12,52.85] \\
2.53[0.12,52.85]\end{array}$ & \\
\hline \multicolumn{8}{|c|}{$\begin{array}{l}\text { Heterogeneity: Not applicable } \\
\text { Test for overall effect: } Z=0.60(P=0.55)\end{array}$} \\
\hline Total $(95 \% \mathrm{Cl})$ & & 44966 & & 30528 & $100.0 \%$ & $0.85[0.75,0.98]$ & 4 \\
\hline Total events & 1498 & & 1135 & & & & \\
\hline $\begin{array}{l}\text { Heterogeneity: } \operatorname{Tau}^{2}=0.02, \\
\text { Test for overall effect: } Z=2.33 \\
\text { Test for subqroup differences: }\end{array}$ & $\begin{array}{l}=17.95, \mathrm{df}=9 \\
(P=0.02) \\
\mathrm{hi}^{2}=1.25, \mathrm{df}=\end{array}$ & $\begin{array}{l}0.04) ; 1^{2}= \\
0.74) \cdot 1^{2}=\end{array}$ & $\begin{array}{l}50 \% \\
=0 \% \\
\end{array}$ & & & & $\begin{array}{ccccccc}1 & 1 & 1 & 1 & 1 & 1 \\
0.1 & 0.2 & 0.5 & 1 & 2 & 5 & 10 \\
& \text { Favours NOA } & \text { Favours Warfarin }\end{array}$ \\
\hline
\end{tabular}

Figure 2 Stroke or systemic embolism random effects model. 
There were six phase II trials of factor Xa inhibitors, edoxaban, betrxiaban, apixaban and YM150 as well as one phase II trial of the direct thrombin (factor IIa) inhibitor AZD 0837. The combined total study population was 77011 . There was one further study identified OPAL-2, which was published as an abstract only with insufficient study methodology and data. Correspondence with the authors did not result in obtaining any further study details.

\section{All stroke and systemic embolism outcomes}

There was significant heterogeneity for the outcome of stroke or systemic embolism the fixed effects model OR 0.87 (95\% CI 0.81 to 0.95 ) suggests a significant reduction in stroke and systemic embolism in the NOACs, this finding was supported by the random effects model OR 0.85 (95\% CI 0.75 to 0.98 ) (figures 1 and 2). There was significant heterogeneity for the outcome of ischaemic stroke OR 1.02 (95\% CI 0.93 to 1.12) suggesting and equivocal result this was finding was supported by the random effects model OR 0.98 (95\% CI 0.82 to 1.16 ) (figure 3). There was a significant reduction in intracranial haemorrhage OR 0.48 (95\% CI 0.0.40 to 0.57 ) (figure 4 ) and mortality OR 0.86 ( $95 \%$ CI 0.82 to 0.91 ) (figure 5 ).

\section{Bleeding outcomes}

There was significant heterogeneity for the composite outcome of major bleeding and non-major clinically relevant bleeding the fixed effects model OR 0.81 (95\% CI 0.77 to 0.84 ) suggests a significant reduction in major bleeding and non-major clinically relevant bleeding in the NOAC's, however, the random effects model showed a non-significant trend towards benefit OR 0.84 (95\% CI 0.68 to 1.03 ). There was significant heterogeneity for the outcome of major bleeding, the fixed effects model OR 0.78 (95\% CI 0.73 to 0.83 ) suggests a significant reduction in major bleeding in the NOAC's, this finding was supported by the random effects model OR 0.76 (95\% CI 0.62 to 0.93). There was significant heterogeneity for the outcome of gastrointestinal bleeding, the fixed effects model OR 1.12 (95\% CI 1.01 to 1.25 ) suggests a significant increase in gastrointestinal bleeding in the NOAC's, however, the random effects model OR 1.05 (95\% CI 0.82 to 1.36) was equivocal.

\section{Myocardial infarction}

There was significant heterogeneity for the outcome of myocardial infarction, the fixed effects model OR 1.02

\begin{tabular}{|c|c|c|c|c|c|c|c|c|}
\hline \multirow[b]{2}{*}{ Study or Subgroup } & \multicolumn{2}{|c|}{ Novel Oral Anticoagulant } & \multicolumn{2}{|c|}{ Warfarin } & \multirow[b]{2}{*}{ Weight } & \multirow{2}{*}{$\begin{array}{l}\text { Odds Ratio } \\
\text { M-H, Random, } 95 \% \mathrm{CI}\end{array}$} & \multirow{2}{*}{\multicolumn{2}{|c|}{$\begin{array}{c}\text { Odds Ratio } \\
\text { M-H, Random, } 95 \% \mathrm{Cl}\end{array}$}} \\
\hline & Events & Total & Events & Total & & & & \\
\hline \multicolumn{9}{|c|}{ 1.2.1 Phase III Trials of F actor Xa Inhibitors } \\
\hline ARISTOTLE 2011 & 162 & 9120 & 175 & 9081 & $16.8 \%$ & $0.92[0.74,1.14]$ & & \\
\hline ROCKET AF 2011 & 149 & 7061 & 161 & 7081 & $16.4 \%$ & $0.93[0.74,1.16]$ & & \\
\hline ENGAGE 2013 Edoxaban 30 & 333 & 7034 & 117 & 3518 & $16.8 \%$ & $1.44[1.17,1.79]$ & & 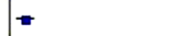 \\
\hline ENGAGE 2013 Edoxaban 60 & 236 & 7035 & 118 & 3518 & $16.4 \%$ & $1.00[0.80,1.25]$ & & \\
\hline $\begin{array}{l}\text { J-Rocket AF } 2012 \\
\text { Subtotal }(95 \% \text { Cl) }\end{array}$ & 7 & $\begin{array}{r}637 \\
30887\end{array}$ & 17 & $\begin{array}{r}637 \\
23835\end{array}$ & $\begin{array}{r}3.2 \% \\
69.6 \%\end{array}$ & $\begin{array}{l}0.41[0.17,0.98] \\
\mathbf{1 . 0 0}[0.79, \mathbf{1 . 2 6}]\end{array}$ & & \\
\hline \multicolumn{9}{|c|}{$\begin{array}{l}\text { Heterogeneity: } \operatorname{Tau}^{2}=0.05 ; \mathrm{Chi}^{2}=15.70, \mathrm{df}=4(\mathrm{P}=0.003) ; \mathrm{I}^{2}=75 \% \\
\text { Test for overall effect: } Z=0.01(P=0.99)\end{array}$} \\
\hline \multicolumn{9}{|c|}{ 1.2.2 Phase III Trials of Direct Thrombin (Factor lla) Inhibitors } \\
\hline RE-LY 2009 Dabigatran 110 & 159 & 6015 & 71 & 3011 & $14.0 \%$ & $1.12[0.85,1.49]$ & & \\
\hline $\begin{array}{l}\text { RE-LY } 2009 \text { Dabigatran } 150 \\
\text { Subtotal }(95 \% \mathrm{CI})\end{array}$ & 111 & $\begin{array}{r}6076 \\
12091\end{array}$ & 71 & $\begin{array}{l}3011 \\
6022\end{array}$ & $\begin{array}{l}13.4 \% \\
27.4 \%\end{array}$ & $\begin{array}{l}0.77[0.57,1.04] \\
0.93[0.65,1.35]\end{array}$ & & \\
\hline \multicolumn{9}{|c|}{$\begin{array}{l}\text { Heterogeneity: } \text { Tau }^{2}=0.05 ; \mathrm{Chi}^{2}=3.21, \mathrm{df}=1(\mathrm{P}=0.07) ; \mathrm{I}^{2}=69 \% \\
\text { Test for owerall effect: } Z=0.36(P=0.72)\end{array}$} \\
\hline \multicolumn{9}{|c|}{ 1.2.3 Phase II Trials of Factor Xa Inhibitors } \\
\hline Explorexa 2013 Betrixaban & 2 & 381 & 0 & 127 & $0.3 \%$ & $1.68[0.08,35.22]$ & & \\
\hline OPAL-1 $2010-Y M 150$ & 1 & 354 & 0 & 94 & $0.3 \%$ & $0.80[0.03,19.85]$ & & \\
\hline Edoxaban USiEurope 2010 & 5 & 893 & 3 & 250 & $1.3 \%$ & $0.46[0.11,1.95]$ & & \\
\hline Edoaban Japan 2012 & 1 & 394 & 0 & 125 & $0.3 \%$ & $0.96[0.04,23.64]$ & & \\
\hline $\begin{array}{l}\text { ARISTOTLEJ } 2011 \\
\text { Subtotal }(95 \% \mathrm{CI})\end{array}$ & 0 & $\begin{array}{r}143 \\
2165\end{array}$ & 2 & $\begin{array}{r}75 \\
671\end{array}$ & $\begin{array}{l}0.3 \% \\
2.5 \%\end{array}$ & $\begin{array}{l}0.10[0.00,2.16] \\
0.52[0.18,1.49]\end{array}$ & & \\
\hline \multicolumn{9}{|c|}{$\begin{array}{l}\text { Heterogeneity: } \operatorname{Tau}^{2}=0.00 ; \mathrm{Chi}^{2}=1.90, \mathrm{df}=4(P=0.75) ;\left.\right|^{2}=0 \% \\
\text { Test for overall effect: } Z=1.22(P=0.22)\end{array}$} \\
\hline \multicolumn{9}{|c|}{ 1.2.4 Phase II Trials of Direct Thrombin (Factor Ila) Inhibitors } \\
\hline $\begin{array}{l}\text { Lip } 2009-A Z D 0837 \\
\text { Subtotal }(95 \% \mathrm{Cl})\end{array}$ & 2 & $\begin{array}{l}361 \\
361\end{array}$ & 1 & $\begin{array}{l}318 \\
318\end{array}$ & $\begin{array}{l}0.5 \% \\
0.5 \%\end{array}$ & $\begin{array}{r}1.77[0.16,19.57] \\
1.77[0.16,19.57]\end{array}$ & & \\
\hline $\begin{array}{l}\text { Total events } \\
\text { Heterogeneity: Not applicable } \\
\text { Test for overall effect: } Z=0.4\end{array}$ & $P=0.64)$ & & 1 & & & & & \\
\hline Total $(95 \% \mathrm{Cl})$ & & 45504 & & 30846 & $100.0 \%$ & $0.98[0.82,1.16]$ & & \\
\hline Total events & 1168 & & 736 & & & & & \\
\hline $\begin{array}{l}\text { Heterogeneity: } \operatorname{Tau}^{2}=0.03, \mathrm{C} \\
\text { Test for owerall effect: } Z=0.2 \\
\text { Test for subgroup differences: }\end{array}$ & $\begin{array}{l}=23.31, \mathrm{df}=1 \\
P=0.77) \\
\mathrm{hi}^{2}=1.68, \mathrm{df}=\end{array}$ & $\begin{array}{l}0.03) ; 1^{2} \\
0.64), 1^{2}\end{array}$ & $\begin{array}{l}=49 \% \\
=0 \%\end{array}$ & & & & $\begin{array}{l}1 \\
0.02 \quad 0.1 \\
\text { Favours NOA }\end{array}$ & Favours Warfarin \\
\hline
\end{tabular}

Figure 3 Ischaemic stroke random effects model. 


\begin{tabular}{|c|c|c|c|c|c|c|c|c|}
\hline \multirow[b]{2}{*}{ Study or Subgroup } & \multicolumn{2}{|c|}{ Novel Oral Anticoagulant } & \multicolumn{2}{|c|}{ Warfarin } & \multirow[b]{2}{*}{ Weight } & \multirow{2}{*}{$\begin{array}{l}\text { Odds Ratio } \\
\text { M-H,Fixed, } 95 \% \mathrm{Cl}\end{array}$} & \multirow{2}{*}{\multicolumn{2}{|c|}{$\begin{array}{c}\text { Odds Ratio } \\
\text { M-H, Fixed, } 95 \% \mathrm{Cl}\end{array}$}} \\
\hline & Events & Total & Events & Total & & & & \\
\hline \multicolumn{9}{|c|}{ 1.3.1 Phase III Trials of F actor Xa Inhibitors } \\
\hline ARISTOTLE 2011 & 40 & 9120 & 78 & 9081 & $22.0 \%$ & $0.51[0.35,0.75]$ & $\varpi$ & \\
\hline ROCKET AF 2011 & 55 & 7111 & 84 & 7125 & $23.5 \%$ & $0.65[0.46,0.92]$ & - & \\
\hline ENGAGE 2013 Edoxaban 30 & 30 & 7034 & 45 & 3518 & $16.9 \%$ & $0.33[0.21,0.53]$ & $\varpi$ & \\
\hline ENGAGE 2013 Edoxaban 60 & 49 & 7035 & 45 & 3518 & $16.8 \%$ & $0.54[0.36,0.81]$ & - & \\
\hline $\begin{array}{l}\text { J-Rocket AF } 2012 \\
\text { Subtotal }(95 \% \text { CI) }\end{array}$ & 5 & $\begin{array}{r}639 \\
30939\end{array}$ & 10 & $\begin{array}{r}639 \\
23881\end{array}$ & $\begin{array}{r}2.8 \% \\
821 \%\end{array}$ & $\begin{array}{l}0.50[0.17,1.46] \\
0.52[0.43,0.63]\end{array}$ & $\dot{1}$ & \\
\hline $\begin{array}{l}\text { Total events } \\
\text { Heterogeneity: } \mathrm{Chi}^{2}=5.44, \mathrm{df}= \\
\text { Test for overall effect: } Z=6.66\end{array}$ & $\begin{array}{r}179 \\
4(P=0.25) ;\left.\right|^{2} \\
P<0.00001)\end{array}$ & & 262 & & & & & \\
\hline \multicolumn{9}{|c|}{ 1.3.2 Phase III Trials of Direct Thrombin (Factor Ila) Inhibitors } \\
\hline RE-LY 2009 Dabigatran 110 & 14 & 6015 & 22 & 3011 & $8.3 \%$ & $0.32[0.16,0.62]$ & . & \\
\hline $\begin{array}{l}\text { RE-LY } 2009 \text { Dabigatran } 150 \\
\text { Subtotal }(95 \% \mathrm{CI})\end{array}$ & 12 & $\begin{array}{r}6076 \\
12091\end{array}$ & 23 & $\begin{array}{r}3011 \\
6022\end{array}$ & $\begin{array}{r}8.7 \% \\
16.9 \%\end{array}$ & $\begin{array}{l}0.26[0.13,0.52] \\
0.29[0.18,0.46]\end{array}$ & & \\
\hline \multicolumn{9}{|c|}{$\begin{array}{l}\text { Heterogeneity: } \mathrm{Chi}^{2}=0.18, \mathrm{df}=1(\mathrm{P}=0.67) ;\left.\right|^{2}=0 \% \\
\text { Test for overall effect: } Z=5.07(P=0.00001)\end{array}$} \\
\hline \multicolumn{9}{|c|}{ 1.3.3 Phase II Trials of Factor Xa Inhibitors } \\
\hline Edoaban Japan 2012 & 1 & 394 & 0 & 125 & $0.2 \%$ & $0.96[0.04,23.64]$ & & \\
\hline OPAL-1 $2010-$ YM150 & 1 & 354 & 0 & 94 & $0.2 \%$ & $0.80[0.03,19.85]$ & & \\
\hline $\begin{array}{l}\text { ARISTOTLEJ } 2011 \\
\text { Subtotal }(95 \% \mathrm{CI})\end{array}$ & 0 & $\begin{array}{l}143 \\
891\end{array}$ & 1 & $\begin{array}{r}75 \\
294\end{array}$ & $\begin{array}{l}0.6 \% \\
1.0 \%\end{array}$ & $\begin{array}{l}0.17[0.01,4.30] \\
\mathbf{0 . 4 8}[0.09,2.60]\end{array}$ & & \\
\hline \multicolumn{9}{|c|}{$\begin{array}{l}\text { Heterogeneity: } C^{2} i^{2}=0.66, d f=2(P=0.72) ; I^{2}=0 \% \\
\text { Test for overall effect: } Z=0.85(P=0.40)\end{array}$} \\
\hline \multicolumn{9}{|c|}{ 1.3.4 Phase II Trials of Direct Thrombin (Factor Ila) Inhibitors } \\
\hline Subtota $(95 \% \mathrm{Cl})$ & & 0 & & 0 & & Not estimable & & \\
\hline $\begin{array}{l}\text { Total events } \\
\text { Heterogeneity: Not applicable } \\
\text { Test for overall effect: Not appl }\end{array}$ & able & & 0 & & & & & \\
\hline Total $(95 \% \mathrm{Cl})$ & & 43921 & & 30197 & $100.0 \%$ & $0.48[0.40,0.57]$ & $\uparrow$ & \\
\hline Total events & 207 & & 308 & & & & & \\
\hline $\begin{array}{l}\text { Heterogeneity: } C \mathrm{Ch}^{2}=11.23 \text {, df } \\
\text { Test for overall effect: } Z=8.10 \\
\text { Test for subqroup differences: }\end{array}$ & $\begin{array}{l}=9(P=0.26) ; I^{\prime} \\
P<0.00001) \\
h i^{2}=5.04, d f=\end{array}$ & 08). $1^{2}$ & $=60.3 \%$ & & & & \begin{tabular}{lc|}
0.01 & 0.1 \\
Favours NOA
\end{tabular} & $1 \frac{1}{10} 100$ \\
\hline
\end{tabular}

Figure 4 Intracranial haemorrhage fixed effects model.

(95\% CI 0.90 to 1.15 ) and the random effects model OR 1.03 (95\% CI 0.88 to 1.20$)$ indicate an equivocal result.

\section{0-day end of study switch to warfarin}

The 30-day end of study switch to warfarin data was only reported by the authors of the ROCKET AF, ENGAGE AF-TIMI 48, ARISTOTLE and J-Rocket AF for the composite outcome of stroke or systemic embolism. The fixed effects model OR 2.60 (95\% CI 1.61 to 4.18 ) is suggestive of significantly more events in the NOAC's arms (figure 6). The 30-day end of study switch to warfarin data was only reported by the authors of the ROCKET AF, ARISTOTLE and ENGAGE AF-TIMI 48 for major bleeding, the fixed effects model OR 2.19 (95\% CI 1.42 to 3.36) suggests significantly more events in the NOAC's arms (figure 7).

Forest plot fixed effects models and where relevant random effects models not presented here are available in the online supplementary appendix figures 5-14.

\section{DISCUSSION}

Twelve studies were identified which were suitable for synthesis of data and meta-analysis, four phase III trials and six phase II trials of factor Xa inhibitors as well as one phase III trial and one phase II trial of direct thrombin (factor IIa) inhibitors with a total study population of 77011 . A further eight supplementary publications providing further insight into the methodology and outcomes were also used to augment the accuracy of this meta-analysis.

All studies included were well conducted randomised controlled trials. Four were double blind trials with a method for double blinding warfarin use. All other studies were of a study design using double blind doses of the study drug in the intervention arm against open label warfarin in the comparison arm. There was one study OPAL-2 that was not included, this was a study of 1297 participants, which data were not sufficiently reported to include.

NOACs when compared to warfarin demonstrated a significant reduction in the composite of stroke or systemic embolic events and were at least as good as warfarin in the prevention ischaemic stroke. There was a notable reduction in intracranial haemorrhage of 52\% with the true population effect of between $60 \%$ and $43 \%$ reduction. This was the main driver of the $14 \%$ reduction in mortality.

Pooled data for the composite of major or clinically relevant non-major bleeding showed significant 


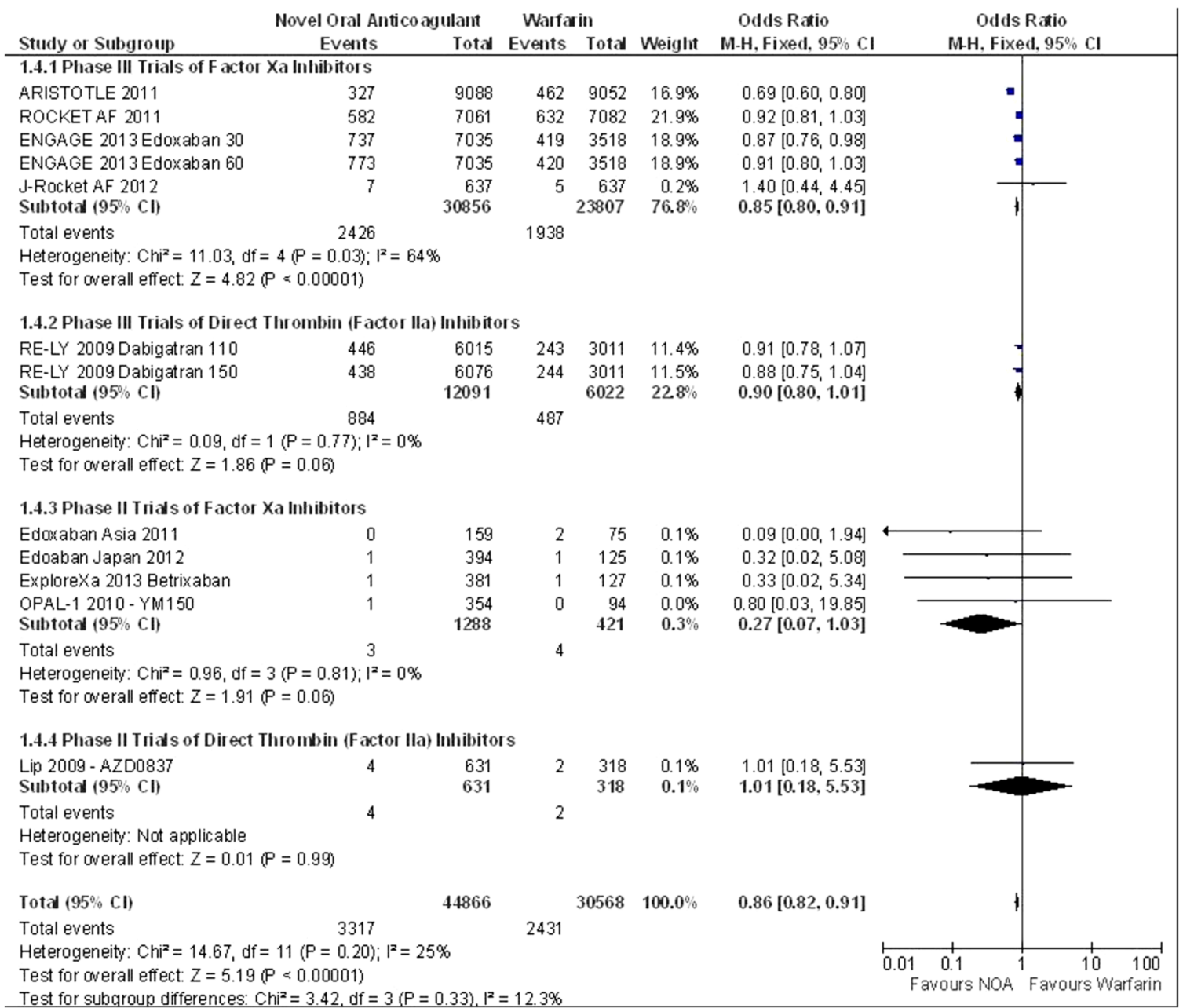

Figure 5 Mortality fixed effects model.

heterogeneity, a fixed effects model indicated that NOACs were superior to warfarin, however, a random effects model indicated a non-significant trend towards benefit. The heterogeneity is likely to have arisen from the ROCKET AF study which included participants of a higher bleeding risk as well as two of the phase II studies, which had multiple study doses, the higher of which were associated with more bleeding events. Indeed the higher twice daily doses of study drugs were noted to achieve higher area under the curve plasma concentrations, with the suggestion of a non-linear relationship between study dose and anticoagulation making higher doses unlikely to be used in phase III trials. There was also a significant reduction in major bleeding with the NOACs, however, phase II studies with higher doses of study drugs were a source of heterogeneity.

Only the phase III trials reported data for gastrointestinal bleeding, pooled data were suggestive of a trend towards less gastrointestinal bleeding in the warfarin arms. There was, however, significant heterogeneity with the ARISTOTLE trial indicating a trend towards benefit

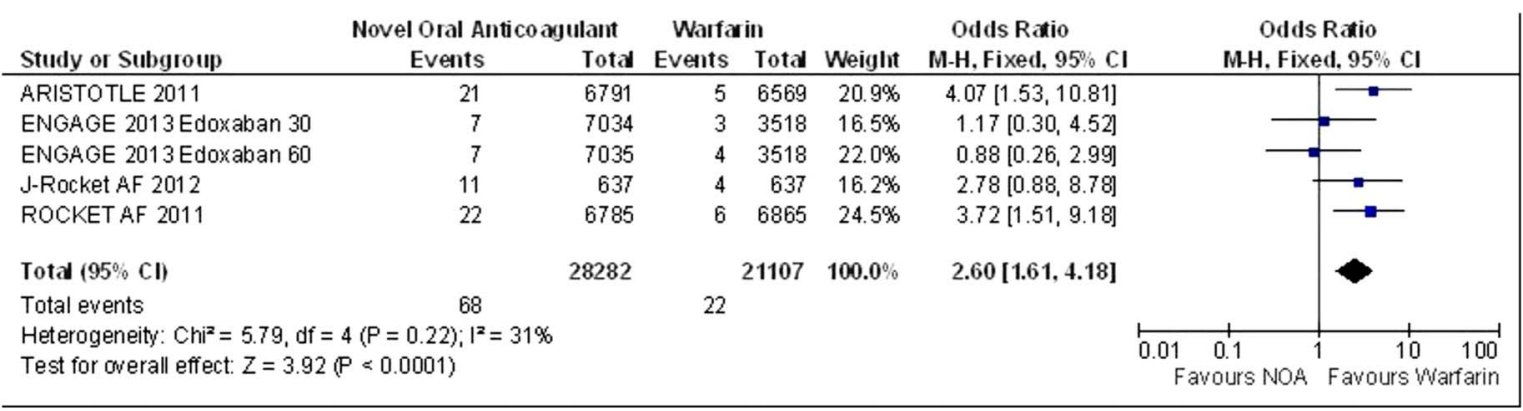

Figure 6 Stroke or systemic embolism 30-day poststudy switch to warfarin fixed effects model. 


\begin{tabular}{|c|c|c|c|c|c|c|c|c|c|}
\hline \multirow[b]{2}{*}{ Study or Subgroup } & \multicolumn{2}{|c|}{ Novel Oral Anticoagulant } & \multicolumn{2}{|c|}{ Warfarin } & \multirow[b]{2}{*}{ Weight } & \multirow{2}{*}{$\begin{array}{l}\text { Odds Ratio } \\
\text { M-H, Fixed, } 95 \% \mathrm{CI}\end{array}$} & \multirow{2}{*}{\multicolumn{3}{|c|}{$\begin{array}{c}\text { Odds Ratio } \\
\text { M-H, Fixed, } 95 \% \text { CI }\end{array}$}} \\
\hline & Events & Total & Events & Total & & & & & \\
\hline ARISTOTLE 2011 & 26 & 6791 & 10 & 6569 & $32.0 \%$ & $2.52[1.21,5.23]$ & & $\because-$ & \\
\hline ENGAGE 2013 Edoxaban 30 & 18 & 7002 & 5 & 3506 & $21.0 \%$ & $1.80[0.67,4.86]$ & & & \\
\hline ENGAGE 2013 Edoxaban 60 & 10 & 7012 & 6 & 3506 & $25.2 \%$ & $0.83[0.30,2.29]$ & & . & \\
\hline ROCKET AF 2011 & 25 & 6785 & 7 & 6865 & $21.9 \%$ & $3.62[1.57,8.38]$ & & $\rightarrow-$ & \\
\hline Total $(95 \% \mathrm{Cl})$ & & 27590 & & 20446 & $100.0 \%$ & $2.19[1.42,3.36]$ & & & \\
\hline Total events & 79 & & 28 & & & & & & \\
\hline $\begin{array}{l}\text { Heterogeneity: } \mathrm{Chi}^{2}=5.17, \mathrm{df}= \\
\text { Test for overall effect: } Z=3.57\end{array}$ & $\begin{array}{l}3(P=0.16) ; 1^{2} \\
P=0.0004)\end{array}$ & & & & & Nowel 0 & $\begin{array}{l}0.01 \quad 0.1 \\
\text { ral Anticoagulant }\end{array}$ & $1_{\text {Warfarin }} 10$ & 100 \\
\hline
\end{tabular}

Figure 7 Major bleeding 30-day poststudy switch to warfarin fixed effects model.

as does the J-ROCKET AF study, which had a slightly lower study dose of $15 \mathrm{mg}$ rivaroxaban once daily rather than the $20 \mathrm{mg}$ once daily dose used in ROCKET AF. There was significantly more gastrointestinal bleeding in ROCKET AF and the higher dose of dabigatran (randomised evaluation of long-term anticoagulant therapy, RE-LY) and a non-significant trend towards harm in the higher dose edoxaban (ENGAGE TIMI AF 48) and lower dose dabigatran (RE-LY). The lower dose edoxaban (ENGAGE TIMI AF 48) showed significantly less gastrointestinal bleeding.

ROCKET AF did include participants with a higher bleeding risk and interestingly the RE-LY trial authors noted that a low $\mathrm{pH}$ is required for absorption of dabigatran and that "dabigatran capsules contain dabigatrancoated pellets with a tartaric acid core." Tartaric acid is found in wine and contributes to its acidity, whether or not this contributes to gastrointestinal bleeding is unclear.

RE-LY did note significantly more dyspepsia in both dabigatran doses than warfarin, which was the most likely adverse reaction leading to discontinuation of the study drug and contributed to the approximate 5\% higher discontinuation of dabigatran compared to warfarin at both 1 and 2 years.

Two of the large phase three studies use two study doses, this could certainly be justified to help understand if particular patient subgroups would benefit from the lower or higher dose as well as the relative effectiveness and safety of each. However, this could also confer the additional advantage when the results are pooled of the benefit of increased anticoagulation and efficacy of the higher dose and the advantageous safety profile of the lower dose.

\begin{tabular}{|c|c|c|c|c|c|c|}
\hline \multicolumn{7}{|c|}{$\begin{array}{l}\text { A systematic review and meta-analysis of the novel oral anticoagulants (NOACs) versus warfarin for the prevention of stroke in patients with atrial fibrillation at an } \\
\text { increased risk of stroke. }\end{array}$} \\
\hline \multicolumn{7}{|c|}{$\begin{array}{l}\text { Patient or population: Patients with atrial fibrillation and an additional risk factor for stroke requiring anticoagulation } \\
\text { Settings: International multicentre clinical trials } \\
\text { Intervention: Novel Oral Anticoagulants (NOA) (Factor Xa and Direct thrombin (factor IIa) inhibitors) } \\
\text { Comparison: Warfarin target INR 2-3 }\end{array}$} \\
\hline \multirow[t]{3}{*}{ Out comes } & \multicolumn{2}{|c|}{ Illustrative comparative risks (95\% CI) } & \multirow{3}{*}{$\begin{array}{l}\text { Relative effect } \\
\text { OR (95\% Cl) }\end{array}$} & \multirow{3}{*}{$\begin{array}{l}\text { No of } \\
\text { Participants } \\
\text { (studies) }\end{array}$} & \multirow{3}{*}{$\begin{array}{l}\text { Quality of the } \\
\text { evidence } \\
\text { (GRADE) }\end{array}$} & \multirow[t]{3}{*}{ Comments } \\
\hline & Assumed risk & Corresponding risk & & & & \\
\hline & Warfarin & Novel Oral Anticoagulants & & & & \\
\hline \multirow[t]{2}{*}{ Stroke or Systemic Embolism } & 37 per 1000 & $\begin{array}{l}32 \text { per } 1000 \\
(30 \text { to } 35)\end{array}$ & \multirow{2}{*}{$\begin{array}{l}\text { OR } 0.85 \\
(0.75 \text { to } 0.98)\end{array}$} & \multirow{2}{*}{$\begin{array}{l}75494 \\
(8)\end{array}$} & ++++ & \\
\hline & 34 per 1000 & $\begin{array}{l}29 \text { per } 1000 \\
\text { (26 to } 33)\end{array}$ & & & High & \\
\hline \multirow{2}{*}{ Ischaemic Stroke } & 24 per 1000 & 23 per 1000 & \multirow{2}{*}{$\begin{array}{l}\text { OR } 0.98 \\
(0.82 \text { to } 1.16)\end{array}$} & \multirow{2}{*}{$\begin{array}{l}76350 \\
(11)\end{array}$} & ++++ & \\
\hline & 23 per 1000 & $\begin{array}{l}(20 \text { to } 28) \\
23 \text { per } 1000 \\
\text { (19 to } 27)\end{array}$ & & & High & \\
\hline \multirow[t]{2}{*}{ Intracranial Haemorrhage } & 10 per 1000 & $\begin{array}{l}5 \text { per } 1000 \\
(4 \text { to } 6)\end{array}$ & \multirow{2}{*}{$\begin{array}{l}\text { OR } 0.48 \\
(0.40 \text { to } 0.57)\end{array}$} & \multirow{2}{*}{$\begin{array}{l}74118 \\
(8)\end{array}$} & ++++ & \\
\hline & 10 per 1000 & $\begin{array}{l}(4 \text { to } 6) \\
5 \text { per } 1000 \\
(4 \text { to } 6 \text { ) }\end{array}$ & & & High & \\
\hline \multirow[t]{2}{*}{ Mortality } & 80 per 1000 & 69 per 1000 & \multirow{2}{*}{$\begin{array}{l}\text { OR } 0.86 \\
(0.82 \text { to } 0.91)\end{array}$} & \multirow{2}{*}{$\begin{array}{l}75434 \\
(10)\end{array}$} & ++++ & \\
\hline & 39 per 1000 & $\begin{array}{l}\text { (66 to } 73) \\
34 \text { per } 1000 \\
\text { (32 to } 36)\end{array}$ & & & High & \\
\hline \multirow{2}{*}{$\begin{array}{l}\text { End of Study } 30 \text { days switch to warfarin } \\
\text { Stroke or systemic embolism }\end{array}$} & 1 per 1000 & 3 per 1000 & \multirow{2}{*}{$\begin{array}{l}\text { OR } 2.60 \\
(1.61 \text { to } 4.18)\end{array}$} & \multirow{2}{*}{$\begin{array}{l}49389 \\
(4)\end{array}$} & +++ & Post hoc data from the \\
\hline & 1 per 1000 & $\begin{array}{l}3 \text { per } 1000 \\
(2 \text { to } 4 \text { ) }\end{array}$ & & & Moderate & $\begin{array}{l}\text { ROCKET AF, ARISTOTLE, } \\
\text { ENGAGE AF-TIMI } 48 \text { and J- } \\
\text { ROCKET AF. }\end{array}$ \\
\hline \multirow{2}{*}{$\begin{array}{l}\text { End of Study } 30 \text { days switch to warfarin } \\
\text { Major bleeding }\end{array}$} & 1 per 1000 & 3 per 1000 & \multirow{2}{*}{$\begin{array}{l}\text { OR } 2.19 \\
\text { (1.42 to } 3.36)\end{array}$} & \multirow{2}{*}{$\begin{array}{l}48036 \\
(3)\end{array}$} & +++ & Post hoc data from the \\
\hline & 2 per 1000 & $\begin{array}{l}(2 \text { to } 5) \\
4 \text { per } 1000 \\
\text { ( } 3 \text { to } 7)\end{array}$ & & & Moderate & $\begin{array}{l}\text { ROCKET AF, ARISTOTLE and } \\
\text { ENGAGE AF-TIMI } 48 \text {. }\end{array}$ \\
\hline
\end{tabular}

(GRADE Working Group grades of evidence 2013)

High quality: Further research is very unlikely to change our confidence in the estimate of effect.

Moderate quality: Further research is likely to have an important impact on our confidence in the estimate of effect and may change the estimate.

Low quality: Further research is very likely to have an important impact on our confidence in the estimate of effect and is likely to change the estimate.

Very low quality: We are very uncertain about the estimate.

Figure 8. Grading of Recommendations Assessment, Development and Evaluation summary of findings table.

Figure 8 Grading of recommendations assessment, development and evaluation summary of findings table. 
Pooled estimates for the outcome of myocardial infarction were equivocal with a point estimate very close to one indicating no associated increase in myocardial infarction with NOACs. The original RE-LY data indicated a significant increase in myocardial infarction for the lower dose of dabigatran, however, this was later revised. ${ }^{20}$ Instead we accepted the amended data, which clearly explained acceptable justification for re-evaluating and blinded assessment of the original trial records. This should be considered in the context that warfarin has the additional incidental benefit of being protective against MI.

There was limited post hoc data for a pooled analysis of stroke and systemic embolism when the studies came to an end and participants taking NOACs were switched to warfarin. During this 30-day switch to warfarin there was a highly significant increase in stroke and systemic embolic events in the NOACs. ROCKET AF reported that the median time to INR within therapeutic range was 13 days for the rivaroxaban arm and 3 days for the warfarin arm, which could elucidate to the increase in embolic events as a return to baseline risk of the warfarin naive patient.

Interestingly the most recent of the large phase III trials ENGAGE TIMI AF 48 did not demonstrate a difference between NOACs and warfarin arms during the 30-day switch and notably had a "comprehensive transition plan to open-label anticoagulation therapy" reported by the authors. Given the short half-life of the NOACs it is certainly plausible that any time between discontinuation and achieving an INR within the therapeutic range is a likely explanation as opposed to a transient prothrombotic state resulting from a rebound effect and highlights the importance of very close monitoring during any transition.

A somewhat surprising finding was the concomitant increase in major bleeding. ARISTOTLE, ROCKET AF and ENGAGE TIMI AF 48 reported major bleeding for the 30-day poststudy switch to warfarin, which showed a significant excess in bleeding events in the NOACs arms. The mechanism by which this could plausibly occur given that the NOACs have a relatively short half-life, would seem to preclude a drug interaction and perhaps may indicate the potentially fluctuant INR levels that can occur during the initiation of warfarin therapy. This again may simply highlight the importance of close clinical supervision during the initiation of warfarin therapy after cessation of long-term NOAC use.

It is, however, unclear whether cessation of long-term therapy for surgical and invasive procedures may show a similar trend and highlights the need for timely cessation of NOACs prior to procedures and vigilant reporting of any thromboembolic or bleeding events.

While warfarin remains an effective drug, the NOACs do confer the additional advantages of fewer interactions with drugs and foods as well as no requirement for routine monitoring, indeed the notion of taking a drug manufactured solely for therapeutic use could also be preferable to taking 'a rat poison'.
Of course warfarin does still retain some advantages, it has been in use for routine clinical practice for many years and is well understood. Testing INR allows for specific tailoring of doses for the individual and perhaps crucially, the administration of vitamin $\mathrm{K}$ effectively reverses warfarin's anticoagulant effect. Conversely the action of NOACs cannot be quickly and effectively reversed, which with their increasing use in clinical practice may leave clinicians almost helpless to intervene when their patients experience incidental trauma.

\section{CONCLUSION}

The NOACs are a safe and effective alternative to warfarin for the prevention of ischaemic stroke in patients with atrial fibrillation and at least one additional risk factor for stroke. The NOACs are at least as good as warfarin for the prevention of ischaemic stroke and systemic embolic events and confers the additional advantage of halving the number of haemorrhagic strokes which drives an overall reduction in mortality. There is also a reduction in major bleeding events associated with the NOACs when compared to warfarin. Cessation of longterm NOAC use and switch to warfarin may be associated with an increase in the composite of ischaemic stroke and systemic embolic events as well as major bleeding in the 30 days after cessation (figure 8), which most likely highlights the necessity of close clinical supervision during this period. Reporting of events during the switch to warfarin in clinical trials is essential in improving understanding of this, as is the reporting of suspected adverse events during transition to warfarin in non-clinical trial settings.

Acknowledgements Revman software was used for meta-analysis, a free download from the Cochrane Collaboration. GRADE pro software was used to formulate the GRADE summary of findings table, a free download from the GRADE working group.

Contributors TH was involved in the study concept, design, screening, statistics and interpretation. FS was involved in the study screening, baseline characteristics and literature search write up. AE was involved in the initial literature search and deduplication of search results.

Competing interests None declared.

Provenance and peer review Not commissioned; externally peer reviewed.

Data sharing statement No additional data are available.

Open Access This is an Open Access article distributed in accordance with the Creative Commons Attribution Non Commercial (CC BY-NC 4.0) license, which permits others to distribute, remix, adapt, build upon this work noncommercially, and license their derivative works on different terms, provided the original work is properly cited and the use is non-commercial. See: http:// creativecommons.org/licenses/by-nc/4.0/

\section{REFERENCES}

1. Go AS, Hylek EM, Chang $Y$, et al. Anticoagulation therapy for stroke prevention in atrial fibrillation: how well do randomized trials translate into clinical practice? JAMA 2003;290:2685-92.

2. Choi HW, Navia JA, Kassab GS. Stroke propensity is increased under atrial fibrillation hemodynamics: a simulation study. PLOS ONE 2013;8:e73485. 
3. Hart RG, Benavente O, McBride R, et al. Antithrombotic therapy to prevent stroke in patients with atrial fibrillation: a meta-analysis. Ann Intern Med 1999;131:492-501.

4. Salazar CA, del Aguila D, Cordova EG. Direct thrombin inhibitors versus vitamin $\mathrm{K}$ antagonists for preventing cerebral or systemic embolism in people with non-valvular atrial fibrillation. Cochrane Database Syst Rev 2014;(3):CD009893.

5. Bruins Slot KM, Berge E. Factor Xa inhibitors versus vitamin $\mathrm{K}$ antagonists for preventing cerebral or systemic embolism in patients with atrial fibrillation. Cochrane Database Syst Rev 2013;(8): CD008980.

6. Mitchell SA, Simon TA, Raza S, et al. The efficacy and safety of oral anticoagulants in warfarin-suitable patients with nonvalvular atrial fibrillation: systematic review and meta-analysis. Clin Appl Thromb Hemost 2013;19:619-31.

7. Hicks $T$, Stewart F, Eisinga A. A systematic review and meta-analysis of the novel oral anticoagulants (NOACs) versus warfarin for the prevention of stroke in patients with atrial fibrillation at an increased risk of stroke. PROSPERO 2014:CRD42014008665 2014. http://www.crd.york.ac.uk/PROSPERO/display_record.asp? ID=CRD42014008665 (accessed 24 Feb 2014).

8. Higgins JP, Thompson SG, Deeks JJ, et al. Measuring inconsistency in meta-analyses. BMJ 2003;327:557-60.

9. Granger $\mathrm{CB}$, Alexander $\mathrm{JH}$, McMurray JJ, et al. Apixaban versus warfarin in patients with atrial fibrillation. $N$ Engl $J$ Med 2011;365:981-92.

10. Lopes RD, Alexander $\mathrm{JH}, \mathrm{Al}-\mathrm{Khatib} \mathrm{SM}$, et al. Apixaban for reduction in stroke and other ThromboemboLic events in atrial fibrillation (ARISTOTLE) trial: design and rationale. Am Heart J 2010;159:331-9.

11. Giugliano RP, Ruff CT, Braunwald E, et al. Edoxaban versus warfarin in patients with atrial fibrillation. $N$ Engl J Med 2013;369:2093-104.

12. Patel MR, Mahaffey KW, Garg J, et al. Rivaroxaban versus warfarin in nonvalvular atrial fibrillation. N Engl J Med 2011;365:883-91.

13. Goodman S, Wojdyla DM, White HD, et al. Predictors of major bleeding risk: insights from the Rivaroxaban Once-daily Oral Direct Factor Xa Inhibition Compared with Vitamin K Antagonism of Stroke and Embolism Trial in Atrial Fibrillation (ROCKET AF). Circulation 2011;124(Suppl.1)

14. Nessel CC, Mahaffey K, Piccini JP, et al. Incidence and outcomes of gastrointestinal hemorrhage in patients with atrial fibrillation treated with rivaroxaban or warfarin: results from the rocket af trial. Chest $J$ 2012;142(Suppl.1):84A.

15. Hori M, Matsumoto M, Tanahashi N, et al., Iwamoto K, Tajiri M; J-ROCKET AF study investigators. Rivaroxaban vs. warfarin in Japanese patients with atrial fibrillation-the J-ROCKET AF study. Circ J 2012;76:2104-11.
16. Connolly SJ, Ezekowitz MD, Yusuf S, et al. Dabigatran versus warfarin in patients with atrial fibrillation. $N$ Engl J Med 2009;361:1139-51.

17. Connolly SJ, Ezekowitz MD, Yusuf S, et al. Newly identified events in the RE-LY trial. N Engl J Med 2010;363:1875-6.

18. Eikelboom JW, Wallentin L, Connolly SJ, et al. Risk of bleeding with 2 doses of dabigatran compared with warfarin in older and younger patients with atrial fibrillation: an analysis of the randomized evaluation of long-term anticoagulant therapy (RE-LY) trial. Circulation 2011:123:2363-72.

19. Hart RG, Diener HC, Yang S, et al. Intracranial hemorrhage in atrial fibrillation patients during anticoagulation with warfarin or dabigatran: the RE-LY trial. Stroke 2012:43:1511-17.

20. Hohnloser SH, Oldgren J, Yang S, et al. Myocardial ischemic events in patients with atrial fibrillation treated with dabigatran or warfarin in the RE-LY (Randomized Evaluation of Long-Term Anticoagulation Therapy) trial. Circulation 2012;125:669-76.

21. Weimar C, Hohnloser SH, Eikelboom JW, et al. Preventing cardioembolic stroke in atrial fibrillation with dabigatran. Curr Neurol Neurosci Rep 2012;12:17-23.

22. Ogawa S, Shinohara $Y$, Kanmuri K. Safety and efficacy of the oral direct factor xa inhibitor apixaban in Japanese patients with non-valvular atrial fibrillation. The ARISTOTLE-J study. Circ $J$ 2011;75:1852-9.

23. Connolly SJ, Eikelboom J, Dorian P, et al. Betrixaban compared with warfarin in patients with atrial fibrillation: results of a phase 2, randomized, dose-ranging study (Explore-Xa). Eur Heart $J$ 2013;34:1498-505.

24. Chung N, Jeon HK, Lien LM, et al. Safety of edoxaban, an oral factor Xa inhibitor, in Asian patients with non-valvular atrial fibrillation. Thromb Haemost 2011;105:535-44.

25. Yamashita T, Koretsune Y, Yasaka M, et al. Randomized, multicenter, warfarin-controlled phase II study of edoxaban in Japanese patients with non-valvular atrial fibrillation. Circ $J$ 2012;76:1840-7.

26. Weitz JI, Connolly SJ, Patel I, et al. Randomised, parallel-group, multicentre, multinational phase 2 study comparing edoxaban, an oral factor Xa inhibitor, with warfarin for stroke prevention in patients with atrial fibrillation. Thromb Haemost 2010;104:633-41.

27. Turpie AGG, Lip GYH, Minematsu K, et al. Safety and tolerability of YM150 in subjects with non-valvular atrial fibrillation: a phase II study. Eur Heart J 2010;31(Suppl 1):173.

28. Lip GY, Rasmussen LH, Olsson SB, et al. Oral direct thrombin inhibitor AZD0837 for the prevention of stroke and systemic embolism in patients with non-valvular atrial fibrillation: a randomized dose-guiding, safety, and tolerability study of four doses of AZD0837 vs. vitamin K antagonists. Eur Heart J 2009;30:2897-907. 